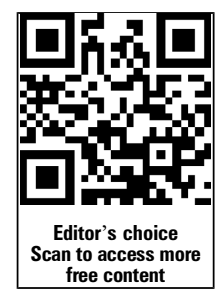

${ }^{1}$ Faculty of Medical and Human Sciences, Institute of Human Development, University of Manchester, Manchester, UK ${ }^{2}$ Manchester Royal Eye Hospital, Manchester, UK ${ }^{3}$ Unit of Health-Care Epidemiology, Nuffield Department of Population Health, University of Oxford, Oxford, UK

\section{Correspondence to}

Tiarnan D L Keenan, Faculty of Medical and Human Sciences, Institute of Human

Development, University of Manchester, AV Hill Building, Oxford Road, Manchester M13 9PT, UK;

tiarnan.keenan@doctors.org.uk

Received 21 July 2014 Revised 17 September 2014 Accepted 30 September 2014 Published Online First 4 November 2014

\title{
Associations between primary open angle glaucoma, Alzheimer's disease and vascular dementia: record linkage study
}

\author{
Tiarnan D L Keenan, ${ }^{1,2}$ Raph Goldacre, ${ }^{3}$ Michael J Goldacre ${ }^{3}$
}

\begin{abstract}
Aims The potential association between primary open angle glaucoma (POAG) and Alzheimer's disease (AD) is uncertain and has implications for understanding disease pathogenesis, referral and treatments. The aim was to determine whether individuals diagnosed with POAG are at higher risk of subsequently developing $A D$ or vascular dementia.

Methods A POAG cohort of 87658 people was constructed from English National Health Service linked hospital episode statistics from 1999 to 2011. An AD cohort (251 703 people), vascular dementia cohort (217 302 people) and reference cohort (>2.5 million people) were constructed in similar ways. Risk of dementia following POAG was determined: rate ratios were calculated based on standardised rates of dementia in the POAG cohort.
\end{abstract}

Results The risk of AD following a diagnosis of POAG was not elevated: the rate ratio was $1.01(95 \% \mathrm{Cl} 0.96$ to 1.06). The risk of vascular dementia after POAG was modestly elevated, with rate ratio 1.10 (1.05 to 1.16 ). The likelihood of a hospital record of POAG following $A D$ or vascular dementia was very low, with rate ratios $0.28(0.24$ to 0.31$)$ and 0.32 (0.28 to 0.37$)$, respectively.

Conclusions POAG and AD are neurodegenerative conditions that share some pathological features. However, considering $A D$ after POAG, their coexistence at the individual level is no different from that expected by chance. By contrast, a diagnosis of POAG is modestly associated with later development of vascular dementia, presumably owing to shared vascular risk factors. People with dementia in England are much less likely to be admitted subsequently with POAG, perhaps through poor access to hospital eye services and diagnostic challenges.

\section{INTRODUCTION}

Primary open angle glaucoma (POAG) and Alzheimer's disease (AD) are neurodegenerative diseases strongly associated with increased age. ${ }^{1}{ }^{2}$ POAG and AD exhibit similarities in some histopathological features: retinal ganglion cells are lost in both conditions, ${ }^{3}$ and hyperphosphorylated $\tau$ is found in the $\mathrm{AD}$ brain and the glaucomatous retina. ${ }^{4}$ In addition, abnormal retinal deposition of amyloid- $\beta$ has been observed in animal models of glaucoma. ${ }^{5}$ However, differences are observed between the two conditions in some of their systemic risk factors; for example, cigarette smoking is thought to increase risk of AD but not of POAG. ${ }^{67}$ The genetic risk factors for the two conditions also appear distinct. The major genetic variant for $\mathrm{AD}$ is at $A P O E$; the $\varepsilon 4$ allele carries higher risk of $\mathrm{AD},{ }^{8}$ but in most studies does not influence POAG risk. ${ }^{9}$

Considerable interest has grown in recent years over the potential relationship between POAG and AD. ${ }^{12}$ This has important implications for understanding disease pathophysiology in the two conditions, and may provide insights into whether existing drugs against targets in one disease (eg, antiamyloid- $\beta$ drugs) may be effective against the other disease. Ongoing laboratory and clinical trials in this area have been reviewed recently, ${ }^{1}$ including strategies based around targeting plaque formation, inflammation, oxidative stress and neuroprotection. In addition, considerable interest has grown in the potential for using retinal changes as a biomarker for AD. ${ }^{1}$ Finally, a positive association might also suggest that patients diagnosed with one condition should be screened for the other.

However, definitive evidence for an epidemiological association has been lacking. Several studies have observed increased prevalence of glaucoma in patients with $\mathrm{AD},{ }^{10}{ }^{11}$ though methodological weaknesses and small study sizes may limit their interpretation. By contrast, other studies have suggested that patients with POAG are not at increased risk of developing AD. ${ }^{12} 13$

We have previously examined the potential association between age-related macular degeneration (AMD) and $\mathrm{AD}$ using record linkage. ${ }^{14}$ Similarly, the main purpose of the current study was to use record linkage to determine whether individuals admitted to hospital with POAG were significantly more or less likely than others to develop $\mathrm{AD}$ in subsequent years. In addition, the risk of developing vascular dementia in subsequent years was examined, given ongoing interest in potential links between POAG and vascular disease..$^{15} 16$

\section{METHODS}

The National Health Service (NHS) provides the majority of healthcare in England, including ophthalmology and medical day-case and inpatient care; this is particularly true for the long-term care of chronic conditions such as dementia and POAG.

As in the previous analysis ${ }^{14}$ the complete data set of linked English national hospital episode statistics (HES), from 1999 to 2011, was used for this study. English national HES are data collected by the NHS Information Centre as a statistical database of demographic, medical and administrative information about all admissions to NHS hospitals in England and admissions funded by the NHS for treatment in non-NHS clinical organisations. Record linkage was undertaken by the Oxford 
record linkage group. Linkage means that data relating to successive episodes of care for each person are brought together, so that data about individuals can be analysed across multiple episodes of care (eg, for POAG and subsequently for $\mathrm{AD}$ ).

A POAG cohort was constructed by identifying all people in the linked HES data set aged 55 years or more who had a record of an admission or day case care where POAG was recorded (International Classification of Diseases Revision 10 (ICD10) code H40.1). For each person, the earliest known record of POAG was the record used in the analysis. A reference cohort was constructed by identifying individuals without POAG who were admitted to hospital for various other, mainly minor medical and surgical, conditions (listed in table 2 footnotes). Unlike the POAG diagnosis, which could be recorded in any diagnostic position on the HES abstract, these conditions were required to be recorded in the main diagnostic position on the hospital record for the individual to be admitted to the reference cohort. People were only included in the POAG or reference cohort if they did not have an admission for $\mathrm{AD}$ or vascular dementia either before or at the same time as the admission with POAG or the reference condition.

We then searched the database for subsequent NHS inpatient or day case care for $\mathrm{AD}$ (ICD10 codes F00, G30) or vascular dementia (ICD10 code F01) in these cohorts. We considered that rates of care for dementia in the reference cohort would approximate those in the general population of the region while allowing for migration in and out of it (data on migration of individuals were not available).

The reverse analysis, that is, POAG hospital diagnoses in people aged 55 years or over with $\mathrm{AD}$ or vascular dementia, was performed using similar methods. In this analysis, people were excluded if they had a record of POAG before, or at the same time as, admission for dementia or reference condition. This ensured that no one was counted twice. The statistical methods used were the same for each analysis. We describe these methods as follows, taking AD in people with POAG as the example.

Rates of people with AD in each cohort were calculated based on person-days 'at risk'. Date of cohort entry was the date of earliest recorded admission for POAG or reference condition, subject to eligibility as described above; date of cohort exit was the date of earliest known record of $\mathrm{AD}$, death or the end of the data collection period (31 December 2011), whichever was the earliest. We adjusted for age in 5-year bands, sex, calendar year of admission, region of residence and Index of Multiple
Deprivation (IMD) score-a standard English measure of socioeconomic status-stratified into quintiles.

Rate ratios were calculated by taking the standardised rate of occurrence of dementia in the POAG cohort relative to the reference cohort. Expected numbers of people with $\mathrm{AD}$ in each cohort were calculated by applying the rates of $\mathrm{AD}$ in the combined population of the POAG and reference cohorts to each individual cohort separately. We did this within each age-sex-year-region-IMD stratum and then summed the stratumspecific expected numbers to obtain expected totals for each cohort. The formula $\left(\mathrm{O}^{\mathrm{POAG}} / \mathrm{E}^{\mathrm{POAG}}\right) /\left(\mathrm{O}^{\mathrm{ref}} / \mathrm{E}^{\mathrm{ref}}\right)$, where $\mathrm{O}$ and $\mathrm{E}$ are the observed and expected numbers of dementia cases in the POAG and reference cohorts, respectively, is equivalent to the rate ratio. The $\mathrm{CI}$ for the rate ratio and $\chi^{2}$ statistics for its significance were calculated as described elsewhere. ${ }^{17}$

\section{RESULTS}

The number of people entering the POAG cohort was 87658 (54\% female); the number of people entering the $\mathrm{AD}$ cohort was 251703 (65\% female), and the number entering the vascular dementia cohort was 217302 (57\% female). The age distributions of each exposure cohort are shown in table 1. More than 2.5 million people entered the reference cohort, providing a plentiful number to populate each age-sex-year-region-IMD stratum of the reference cohort.

For people with POAG, there was no significant association with $\mathrm{AD}$ : the rate ratio, comparing the POAG cohort with the reference cohort, was 1.01 (95\% CI 0.96 to 1.06). There was a small but statistically significant increase in the risk of vascular dementia: the rate ratio was 1.10 (95\% CI 1.05 to 1.16) (table 2).

People in the $\mathrm{AD}$ cohort and the vascular dementia cohort were very significantly less likely than those in the reference cohort to receive a subsequent record of POAG: the rate ratio, comparing the $\mathrm{AD}$ cohort with the reference cohort, was 0.28 (0.24 to 0.31$)$; comparing the vascular dementia cohort with the reference cohort, the rate ratio was 0.32 (0.28 to 0.37) (table 3).

\section{DISCUSSION}

These data support the hypothesis that individuals with POAG are not at increased risk of subsequently developing $\mathrm{AD}$. However, a modest but significant positive association was found between POAG and vascular dementia.

As mentioned above, previous studies have reported varying results for a potential association between POAG and $\mathrm{AD}$,

Table 1 The number $(N)$ and age-sex distribution of the people who entered the primary open angle glaucoma $(P O A G)^{*}$ cohort, the Alzheimer's disease $(A D)+$ cohort, the vascular dementiał cohort, and the reference cohort

\begin{tabular}{|c|c|c|c|c|c|c|c|c|c|c|c|c|}
\hline \multirow[b]{2}{*}{ Age } & \multicolumn{3}{|c|}{ POAG cohort } & \multicolumn{3}{|c|}{ AD cohort } & \multicolumn{3}{|c|}{ Vascular dementia cohort } & \multicolumn{3}{|c|}{ Reference cohort } \\
\hline & $\mathrm{N}$ & (\% of total) & $\%$ female & N & N & (\% of total) & $\%$ female & (\% of total) & $\%$ female & N & ( $\%$ of total) & $\%$ female \\
\hline $55-59$ & 3116 & (3.6) & 44.4 & 2127 & 1037 & $(0.5)$ & 33.3 & $(0.8)$ & 54.2 & 481856 & $(19.0)$ & 49.2 \\
\hline $60-64$ & 5148 & (5.9) & 46.6 & 4444 & 2839 & (1.3) & 35.3 & $(1.8)$ & 52.7 & 484001 & $(19.1)$ & 47.6 \\
\hline $65-69$ & 8308 & $(9.5)$ & 48.5 & 9224 & 7682 & (3.5) & 38.4 & (3.7) & 54.4 & 448135 & (17.7) & 47.7 \\
\hline 70-74 & 13082 & $(14.9)$ & 50.4 & 22325 & 18698 & (8.6) & 43.7 & (8.9) & 56.8 & 412589 & $(16.3)$ & 48.8 \\
\hline 75-79 & 19199 & (21.9) & 52.5 & 48295 & 39786 & $(18.3)$ & 49.5 & $(19.2)$ & 60.2 & 346924 & (13.7) & 50.7 \\
\hline $80-84$ & 20061 & (22.9) & 56.2 & 68029 & 58187 & (26.8) & 56.2 & (27) & 64.6 & 218801 & (8.6) & 53.7 \\
\hline $85+$ & 18744 & (21.4) & 62.3 & 97259 & 89073 & (41) & 66.0 & (38.6) & 71.0 & 143166 & (5.6) & 60.0 \\
\hline Total & 87658 & $(100)$ & 54.1 & 251703 & 217302 & (100) & 56.9 & (100) & 64.9 & 2535472 & $(100)$ & 49.8 \\
\hline
\end{tabular}


Table 2 Observed (0) and expected (E) numbers of people in the primary open angle glaucoma cohort who received a subsequent hospital record of Alzheimer's disease (AD) or vascular dementia; observed $\left(\mathrm{O}^{\text {ref }}\right)$ and expected $\left(\mathrm{E}^{\text {ref }}\right)$ numbers of people in the reference cohort* who received a subsequent hospital record of $\mathrm{AD}$ or vascular dementia; standardised rate ratios (RR) and $95 \%$ $95 \% \mathrm{Cls}$

\begin{tabular}{llllll}
\hline Outcome & $\mathbf{0}$ & $\mathbf{E}$ & $\mathbf{0}^{\text {ref }}$ & $\mathbf{E}^{\text {ref }}$ & $\mathbf{R R}(95 \% \mathrm{Cl})$ \\
\hline AD & 1701 & 1686.6 & 28997 & 29011.4 & $1.01(0.96$ to 1.06) \\
Vascular dementia & 1844 & 1681.3 & 27676 & 27838.7 & $1.10(1.05$ to 1.16)
\end{tabular}

*The reference cohort consisted of people admitted with the following conditions coded under ICD-10 for diagnoses and Office of Population Censuses and Surveys Classification of Interventions and Procedures version 4 (OPCS-4) for operations: otitis externa/media (ICD-10 H60-H67), varicose veins (I83), haemorrhoids (I84), upper respiratory tract infections (J00-J06), deflected septum, nasal polyp (J33+J34.2), impacted tooth and other disorders of teeth (K00-K03), inguinal hernia (K40), gall bladder disease (K80-K81), ingrowing toenail and other diseases of nail (L60), sebaceous cyst (L72.1), bunion (M20.1), appendectomy (OPCS-4 H01-H03), dilation and curettage (Q10.3-Q10.4), total hip replacement (W37-W39), total knee replacement (W40-W42).

dementia or cognitive impairment. ${ }^{10-13} 1819$ In 2002, Bayer et $a l^{10}$ reported a higher prevalence of glaucoma (26\%) in 112 individuals with probable AD living in nursing homes, compared with $5 \%$ in a control group. However, the authors acknowledged that the study was limited by potential selection bias, case-control design and lack of blinding in the observers. In addition, it is possible that POAG may be overdiagnosed in patients with dementia, as accuracy of perimetry and ophthalmological examination may be problematic. Tamura et al ${ }^{11}$ conducted a case-control study in Japan, involving 172 patients with $\mathrm{AD}$ and 176 age-matched controls, and reported a higher prevalence of open angle glaucoma $(\mathrm{OAG})$ in the $\mathrm{AD}$ group ( $24 \%$ vs $10 \%)$. However, this study is limited by its design and size, and these findings may not be applicable to other ethnic populations, as normal tension glaucoma (NTG) makes up as much as $90 \%$ of OAG in Japan. ${ }^{20}$

Our findings are consistent with previous smaller studies suggesting that patients with POAG are not at increased risk of developing AD. ${ }^{12} 13$ Kessing et $a l^{12}$ used a record linkage study design to analyse the risk of subsequent $\mathrm{AD}$ in 11721 patients with POAG (including NTG) in Denmark, and reported no increased risk compared with the general population; the rate ratio was 0.76 , but with relatively wide $95 \%$ CI (0.56 to 1.05$)$. In addition, a large retrospective study of Medicare recipient data in the USA was conducted by Ou et al. ${ }^{13}$ Consistent with our study, this analysis reported no increased risk of subsequent $\mathrm{AD}$ in patients with $\mathrm{OAG}$.

One further study performed testing for cognitive impairment on elderly individuals with and without glaucoma in the UK. ${ }^{19}$

Table 3 Observed (O) and expected (E) numbers of people in the Alzheimer's disease (AD) and vascular dementia cohorts who received a subsequent hospital record of primary open angle glaucoma (POAG); observed $\left(0^{\text {ref }}\right)$ and expected $\left(\mathrm{E}^{\text {ref }}\right)$ numbers of people in the reference cohort who received a subsequent hospital record of POAG; standardised rate ratios (RR) and $95 \% \mathrm{Cls}$

\begin{tabular}{llllll}
\hline Cohort & $\mathbf{0}$ & $\mathbf{E}$ & $\mathbf{0}^{\text {ref }}$ & $\mathbf{E}^{\text {ref }}$ & RR $(95 \% \mathrm{Cl})$ \\
\hline AD & 222 & 769.7 & 12230 & 11682.3 & $0.28(0.24$ to 0.31$)$ \\
Vascular dementia & 203 & 604.4 & 12174 & 11772.6 & $0.32(0.28$ to 0.37$)$ \\
\hline
\end{tabular}

The OR for those with glaucoma having a lower score on the standardised mini-mental state examination (MMSE) was 1.76 (1.05 to 2.95). However, when using the 'blind' version of the MMSE (which omits several vision-dependent tasks of the standard MMSE), the OR for a lower score was only 1.17 (0.65 to 2.12). Importantly, this suggests that any apparent relationship between glaucoma and cognitive impairment (based on standard MMSE tests) may be artefactual. This caveat might also apply to a study in France, which reported that participants with OAG had increased risk of dementia (OR 3.9, 1.5 to 10.4). ${ }^{18}$ While this study had methodological strengths in its longitudinal design and active screening for OAG and dementia, it was limited by its small size (only 41 cases of dementia), short follow-up period ( 3 years) and inability to analyse according to type of dementia.

The potential relationship between POAG and vascular dementia is interesting, and we are unaware of any previous published reports of this association. Possible explanations for this weakly positive association may include the presence of systemic risk factors (excluding the ones controlled for in this study) that are shared between POAG and vascular dementia. Vascular risk factors are likely to be important in this context. For example, diabetes mellitus and systemic hypertension are strong risk factors for vascular dementia, ${ }^{21} 22$ and may also increase the risk of POAG. ${ }^{23}$ In addition, previous research has demonstrated that patients with OAG have reduced cerebrovascular blood flow and vasoreactivity compared with controls, ${ }^{24}$ and that patients with NTG in particular have a high incidence of macrovascular and microvascular abnormalities. ${ }^{16}$

The other main finding in our study was that individuals with dementia were substantially less likely to have subsequent hospital admissions with recorded POAG. We recently reported a similar finding for patients with dementia undergoing treatment for AMD. ${ }^{14}$ As discussed previously for AMD treatment, this might represent a genuine deficit in ophthalmic care for people with dementia. This may be particularly true for glaucoma, since POAG usually presents insidiously without pain or initial visual symptoms. Interestingly, dementia and cognitive impairment appear not to have been considered in previous analyses of potential risk factors for late presentation of OAG. ${ }^{25}$

We have previously considered potential reasons why individuals with dementia may suffer a deficit in care for ophthalmic conditions, ${ }^{14}$ and similar considerations apply to POAG. These include being less likely to attend regular optometrist appointments, being less likely to notice or report visual symptoms, and being reliant on carers to act on reported symptoms. However, there are few reports in the literature examining these factors, and we are unaware of recommendations or guidelines published by national ophthalmology or neurology bodies that deal with provision of glaucoma services for patients with dementia. Further research across a variety of countries is required to determine whether people with dementia are receiving appropriate investigation and treatment for glaucoma, and to identify and address any potential barriers to care.

Finally, it is also possible that the low rate ratio might reflect a genuine negative association between dementia and POAG (occurring in that order). For example, medical control of vascular risk factors following a diagnosis of vascular dementia (or even $\mathrm{AD}$ ) could potentially decrease the risk of POAG or slow its progression. It is also conceivable that medications prescribed for $\mathrm{AD}$ (eg, N-methyl-D-aspartic acid (NMDA) antagonists) might have had a shared neuroprotective effect in preserving retinal ganglion cells and decreasing POAG risk. While this would be of great interest, our study could not distinguish 
between these possibilities, and further research may be warranted.

The strengths of this study include the very large size of the data set, the completeness of the routinely collected data, and the long period of follow-up. These factors provide substantial improvements on some previous studies. We have also used this data set in previous studies of ophthalmic disease associations. ${ }^{14}$ Through record linkage we were able to carry out several cohort analyses to examine the rates of dementia in people with POAG, as well as the rates of POAG in people with dementia, and conducted separate analyses for $\mathrm{AD}$ and vascular dementia.

However, the data set has limitations. The data are based on hospital admissions and day case specialist care for the particular conditions, and are therefore likely to represent the more severe end of the spectrum of disease. However, for POAG, individuals undergoing day case or inpatient care for any reason were included in the POAG cohort, if POAG was documented on the admission record. In addition, all patients undergoing laser treatment or surgery for POAG were included, as in the English NHS, these procedures generate an admission record. Nevertheless, this methodology will not capture all patients with POAG in England, particularly those treated over a long period exclusively with medical therapy, and who are not admitted over the study period for other ophthalmic (eg, cataract surgery) or medical/surgical conditions.

Other limitations with this data set have been described previously, for example, reliability of HES coding for $\mathrm{AD}$ versus other dementia; cohorts based on prevalent cases; unmeasured migration; and possible confounding factors such as cigarette smoking or other cardiovascular risk factors. ${ }^{14}$ In addition, we were not able to discriminate in this study between NTG and POAG, as the ICD-10 code H40.1 does not make this distinction.

In conclusion, these data provide evidence that there is no positive association between POAG and AD. However, a weakly positive association was found between POAG and vascular dementia. Vascular risk factors may be responsible for this relationship, particularly in the context of NTG. People with dementia in England are substantially less likely to receive a subsequent hospital diagnosis of POAG. Any potential deficit in glaucoma care for these vulnerable individuals needs to be examined and addressed in the near future.

Acknowledgements Over many years, the linked data files, and associated analytical software, were built by Leicester Gill, Matt Davidson and David Yeates, Unit of Health-Care Epidemiology, University of Oxford.

Contributors Design and conduct of the study; collection, management, analysis and interpretation of the data; review and appraisal of the manuscript: TDLK, RG, MJG; preparation: TDLK.

Funding The Unit of Health-Care Epidemiology was funded by the English National Institute for Health Research to build the linked data set and to make it available for analysis. This study had no specific funding. TDLK is funded by a Fulbright/Fight for Sight UK-US Scholarship. The views expressed in this paper do not necessarily reflect those of the funding bodies.

Competing interests None.
Ethics approval Ethics Committee approval for analysis of the record linkage study data was obtained from the Central and South Bristol Multi-Centre Research Ethics Committee, England (04/Q2006/176). The research adhered to the tenets of the Declaration of Helsinki.

Provenance and peer review Not commissioned; externally peer reviewed.

\section{REFERENCES}

1 Sivak JM. The aging eye: common degenerative mechanisms between the Alzheimer's brain and retinal disease. Invest Ophthalmol Vis Sci 2013;54:871-80.

2 Ghiso JA, Doudevski I, Ritch R, et al. Alzheimer's disease and glaucoma: mechanistic similarities and differences. J Glaucoma 2013;22(Suppl 5):S36-8.

3 Blanks JC, Torigoe Y, Hinton DR, et al. Retinal pathology in Alzheimer's disease. I. Ganglion cell loss in foveal/parafoveal retina. Neurobiol Aging 1996; 17:377-84.

4 Noble W, Hanger DP, Miller CC, et al. The importance of tau phosphorylation for neurodegenerative diseases. Front Neurol 2013;4:83.

5 Kipfer-Kauer A, McKinnon SJ, Frueh BE, et al. Distribution of amyloid precursor protein and amyloid-beta in ocular hypertensive C57BL/6 mouse eyes. Curr Eye Res 2010;35:828-34.

6 Cataldo JK, Prochaska JJ, Glantz SA. Cigarette smoking is a risk factor for Alzheimer's Disease: an analysis controlling for tobacco industry affiliation. J Alzheimers Dis 2010;19:465-80.

7 Ramdas WD, Wolfs RC, Hofman A, et al. Lifestyle and risk of developing open-angle glaucoma: the Rotterdam study. Arch Ophthalmol 2011;129:767-72.

8 Medway C, Morgan K. Review: the genetics of Alzheimer's disease; putting flesh on the bones. Neuropathol Appl Neurobiol 2014;40:97-105.

9 Zetterberg M, Tasa G, Palmer MS, et al. Apolipoprotein E polymorphisms in patients with primary open-angle glaucoma. Am J Ophthalmol 2007;143:1059-60.

10 Bayer AU, Ferrari F, Erb C. High occurrence rate of glaucoma among patients with Alzheimer's disease. Eur Neurol 2002;47:165-8.

11 Tamura $\mathrm{H}$, Kawakami $\mathrm{H}$, Kanamoto $\mathrm{T}$, et al. High frequency of open-angle glaucoma in Japanese patients with Alzheimer's disease. J Neurol Sci 2006;246:79-83.

12 Kessing LV, Lopez AG, Andersen PK, et al. No increased risk of developing Alzheimer disease in patients with glaucoma. J Glaucoma 2007;16:47-51.

13 Ou Y, Grossman DS, Lee PP, et al. Glaucoma, Alzheimer disease and other dementia: a longitudinal analysis. Ophthalmic Epidemiol 2012;19:285-92.

14 Keenan TD, Goldacre R, Goldacre MJ. Associations between age-related macular degeneration, Alzheimer disease, and dementia: record linkage study of hospital admissions. JAMA Ophthalmol 2014;132:63-8.

15 Kang JH, Loomis SJ, Yaspan BL, et al. Vascular tone pathway polymorphisms in relation to primary open-angle glaucoma. Eye (Lond) 2014;28:662-71.

16 Mroczkowska S, Ekart A, Sung V, et al. Coexistence of macro- and micro-vascular abnormalities in newly diagnosed normal tension glaucoma patients. Acta Ophthalmol 2012;90:e553-9.

17 Breslow NE, Day NE. Statistical methods in cancer research. Volume II-The design and analysis of cohort studies. IARC Sci Pub/ 1987;82:1-406.

18 Helmer $C$, Malet F, Rougier MB, et al. Is there a link between open-angle glaucoma and dementia?: the Three-City-Alienor Cohort. Ann Neurol 2013;74:171-9.

19 Jefferis JM, Taylor JP, Collerton J, et al. The association between diagnosed glaucoma and cataract and cognitive performance in very old people:cross-sectional findings from the newcastle 85+ study. Ophthalmic Epidemiol 2013;20:82-8.

20 Iwase A, Suzuki Y, Araie M, et al. The prevalence of primary open-angle glaucoma in Japanese: the Tajimi Study. Ophthalmology 2004;111:1641-8.

21 ladecola C. Hypertension and dementia. Hypertension 2014;64:3-5.

22 Ninomiya T. Diabetes mellitus and dementia. Curr Diab Rep 2014;14:487.

23 Janssen SF, Gorgels TG, Ramdas WD, et al. The vast complexity of primary open angle glaucoma: disease genes, risks, molecular mechanisms and pathobiology. Prog Retin Eye Res 2013;37:31-67.

24 Zhang S, Xie Y, Yang J, et al. Reduced cerebrovascular reactivity in posterior cerebral arteries in patients with primary open-angle glaucoma. Ophthalmology 2013;120:2501-7.

25 Deva NC, Insull E, Gamble G, et al. Risk factors for first presentation of glaucoma with significant visual field loss. Clin Experiment Ophthalmol 2008;36:217-21. 